\title{
Scanning transmission electron microscopy assessment of a metal-organic framework compound
}

Peter Moeck $^{1}$, B. Layla Mehdi ${ }^{2}$, Andrew Stevens ${ }^{3}$, Paul DeStefano ${ }^{1}$, Nigel Browning ${ }^{2}$

${ }^{1}$ Portland State University, Portland, United States, ${ }^{2}$ Physical and Computational Science Directorate, Pacific Northwest National Laboratory, Richland, WA 99352, United States, ${ }^{3}$ National Security Directorate, Pacific Northwest National Laboratory, Richland, WA 99352, United States

E-mail: pmoeck@pdx.edu

Discrete (and fast) Fourier transforms (FTs) of a low-dose high-angle annular dark-field image that was recorded from a metal-organic framework (MOF) compound with a probe-corrected scanning transmission electron microscope (STEM) along the [0001] direction revealed weak spots that violate the 2D space group symmetry one would expect on the basis of this material's published single-crystal X-ray crystallography structure [1]. These spots are consistent with the general and special reflection conditions of an approximately twice as large projected unit cell with much reduced pseudo-symmetry on a pseudo-rectangular lattice with an $\mathrm{a} / \mathrm{b}$ ratio close to the inverse of the square root of three. Our preliminary explanation for these spots is long-range positional order at the center of the meso-scopic channels of parts of this MOF, which the X-ray crystallography structure claims to be devoid of matter with such order. Fourier filtered and cystallographically processed direct space STEM images with much improved signal to noise ratios support this explanation. One needs, however, to be aware that a STEM image is just a projection of the samples structure into two dimensions (2D) if no attempt at a tomographic reconstruction has been made. (Due to the very high sensitivity of MOFs to the electron beam, a tomographic reconstruction may not be possible.) Locally varying average 2D lattice parameters were determined by calculating FTs from adjacent areas of STEM images (that were corrected for scanning distortions) from the same batch of MOF. All 2D lattices were oblique but either pseudo-hexagonal or pseudo-rectangular. The detected locally varying lattice parameters may be a consequence of local differences in the fillings of the meso-scopic channels. Structural defects in the form of additional transition metal containing nodes at slightly different positions within the meso-scopic channels were also visible in raw STEM images from other areas of this MOF despite the low signal to noise ratio. The MOF material may, therefore, not be crystalline in the traditional sense [2]. Note that there is already precedence in another MOF for this kind of deviation from traditional crystalline ideal structures [3], as recently demonstrated with synchrotron based powder X-ray diffraction analyses on the basis of the Debye scattering equation. While we are well aware that all published X-ray crystallography structures are idealizations and result from averaging over a very large amount of unit cells, we consider our results worthwhile publishing because a part of the long-range ordered structure that should have consequences for the physical and chemical properties of this material has so far not been reported. Our studies want to draw attention to this omission, describe the missing part of the ideal structure qualitatively and reveal structural defects in this material. Hopefully, this will spur more structural investigations of MOFs by complementing direct and reciprocal space methods.

[1] see http://www.crystallography.net/cod/4120127.html at the Crystallography Open Database for an interactive visualization. [2] Hosemann R. \& Hindeleh, A. M. (1995) J. Macromol. Science - Physics B 34, 327-356. [3] Mason, J. A. et al. (2015) Nature 527, 357-361.

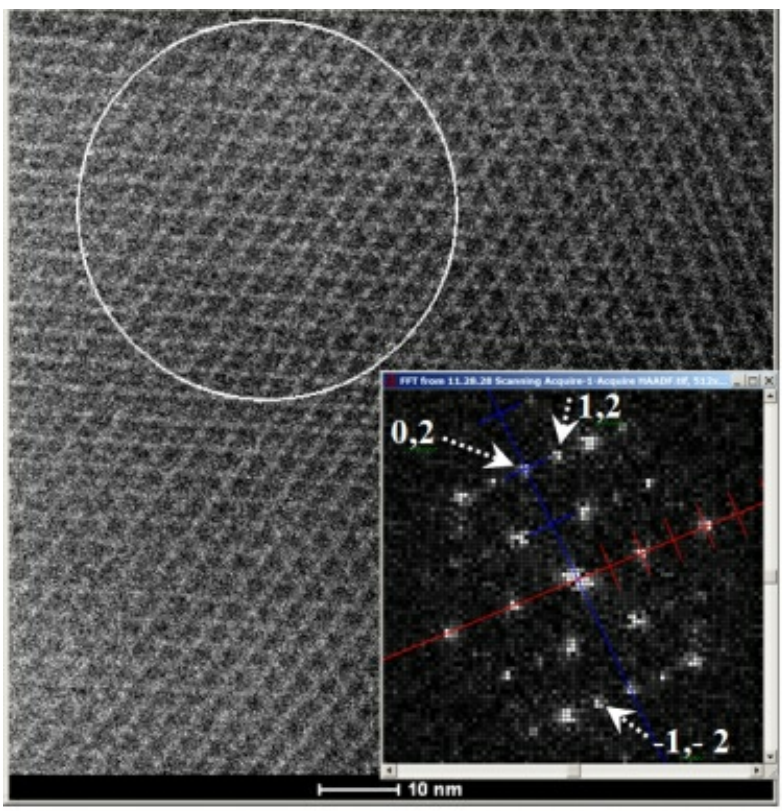

Keywords: $\underline{\text { metal-organic framework, STEM imaging, defects }}$ 